\title{
Inflatable Microstrip Reflectarray Antennas At $X$ and Ka-Band Frequencies
}

\author{
John Huang and Alfonso Feria \\ Jet Propulsion Laboratory \\ California Institute of technology \\ Pasadena, CA, 91109, USA
}

Introduction: Inflatable antenna technology is being developed by JPL/NASA to enable the capabilities of low mass, high packaging efficiency, and low-cost deployment for future spacecraft high-gain and large aperture antennas. One of the technologies being considered [1] is the inflatable microstrip reflectarray. A conventional inflatable parabolic reflector antenna will offer similar advantages with the added capability of wide electrical bandwidth. However, it suffers from the difficulty of maintaining its required large, thin, and curved-parabolic surface in the space environment. Since the microstrip reflectarray $[2,3,4,5]$ has the "natural" flat reflecting surface, it is much easier to maintain the required surface tolerance using an inflatable structure. This is the primary reason, despite its narrow bandwidth characteristic, that the inflatable microstrip reflectarray is being studied. This article discusses an already-developed one-meter $\mathrm{X}$-band inflatable microstrip reflectarray and a three-meter Ka-band inflatable microstrip reflectarray which is currently under development. Both antennas' RF structures are designed at JPL and their mechanical inflatable structures are designed and manufactured at ILC Dover, Inc.

Description of the One-Meter X-band Antenna: A photograph of the circularly polarized Xband inflatable microstrip reflectarray with a one-meter aperture is shown in Fig. 1. The antenna consists of three primary components: a one-meter-diameter thin-membrane reflectarray surface, an inflatable feed-support tripod structure, and a circular inflatable torus that supports the thinmembrane reflectarray surface. Both the feed tripod and the torus are made of inflatable tube 13 $\mathrm{cm}$ in diameter fabricated using $0.25-\mathrm{mm}$ thick urethane coated kevlar material. Because of the possibility that the structure may lose pressure after initial inflation due to leakage (micrometeorite penetration, manufacturing defects, etc.), the material would need to be rigidized in order to maintain its structural integrity. Several rigidizing techniques and materials (stretched aluminum, hydro-gel based material, thermally controlled epoxy, etc.) are being studied for long-term space missions. The one-meter reflectarray surface consists of two layers of thin membranes with each layer made of 5 -micron thick copper deposited on 0.05 -mm thick Kapton membrane. The copper on the top layer was etched to form approximately one thousand microstrip patches (each $1.5 \mathrm{~cm}$ square) with different-length phase delay lines attached $[2,3,4]$ as illustrated in Fig. 2. Each patch is dual-linear polarized with two orthogonal phase delay lines attached. With the feed circularly polarized, the reflectarray radiates circular polarization. The bottom layer serves as ground plane and is separated $1.3 \mathrm{~mm}$ from the top layer by several hundred small circular foam disks. With this configuration, the microstrip patches are essentially over an air substrate. The low-mass foam disks, $7-\mathrm{mm}$ diameter each, are used to maintain a uniform membrane spacing after the deployment of the inflatable structure so that all patches resonate at the same $R F$ frequency. The two-layer reflectarray membrane surface is supported to maintain its required flatness by the inflatable torus at 15 circumferential catenary attachment 
points. The tension of the attachment points can be adjusted to stretch the membranes to achieve the required surface flatness. The $1-\mathrm{m}$ reflectarray surface achieved a flatness tolerance of less than $\pm 1.3 \mathrm{~mm}(1 / 20$ wavelength is $1.8 \mathrm{~mm})$. Excluding the mass of the inflation system and the feed, the inflatable reflectarray achieved a mass of $1.2 \mathrm{~kg}$.

The antenna pattern measured at $8.3 \mathrm{GHz}$ is shown in Fig. 3. It has a $3-\mathrm{dB}$ beamwidth of $2.4^{\circ}$, a peak sidelobe level of $-18 \mathrm{~dB}$, and a peak cross-pol level of $-18 \mathrm{~dB}$. The beamwidth is as expected for a one-meter aperture. The sidelobe level of $-18 \mathrm{~dB}$, although is acceptable to the telecom system, is higher than the expected $-25 \mathrm{~dB}$ level. This relatively high sidelobe level is believed to be the result of feed and strut blockages, imperfect membrane separation, and a certain amount of surface roughness. The higher-than-expected cross-pol level $(-18 \mathrm{~dB}$ rather than $-25 \mathrm{~dB}$ ) is believed to be caused by the phase delay lines attached to the patches. The cross-pol fields from the feed horn and from all the patches are all coherently directed in the main beam direction as the co-pol fields by the same set of phase delay lines [6]. This is why a conventional reflectarray generally has a high cross-pol beam situated in the co-pol's main beam direction. The measured antenna gain peaked at $8.3 \mathrm{GHz}$ and is $33.7 \mathrm{~dB}$ which yields an overall antenna efficiency of $37 \%$, while the expected efficiency is about $50 \%$. This relatively poor efficiency is primarily due to imperfect membrane separation (between patches and the ground plane). This error is believed to be correctable by a better mechanical design and a more careful manufacturing process.

Description of the Three-Meter Ka-Band Antenna: A schematic drawing of the 3-m Ka-band inflatable microstrip reflectarray is shown in Fig. 4. Similar to the $1-\mathrm{m} \mathrm{X}$-band antenna, it consists of three major components: a 3-m-diameter RF aperture thin-membrane surface, an asymmetrical inflatable feed-strut tripod structure, and a horseshoe-like inflatable torus that supports and tensions the 3-m thin-membrane surface. Both the feed tripod and the torus are made of inflatable tubes with $0.25 \mathrm{~mm}$ thick urethane coated Kevlar material. The torus tube has a diameter about $25 \mathrm{~cm}$, which is determined by the stretching force needed to maintain an appropriate membrane flatness. The top of the horseshoe-like torus is a straight non-inflatable rigid tube made of low-mass composite material. With the tripod tubes asymmetrically placed at the lower portion of the torus, the rigid composite tube can roll up the RF membrane surface to a stowed configuration without causing significant wrinkles and unevenness to the membrane surface. At the Ka-band frequency, maintaining the required surface tolerance $( \pm 0.5 \mathrm{~mm})$ is the primary concern for this 3-m aperture antenna. The 3-m diameter reflectarray surface is a singlelayer membrane having a 0.13 -mm-thick substrate made of polyimide material with 5 -micron copper deposited on both sides. On one side, the copper is etched to form approximately 200,000 patch elements. Unlike the $1-\mathrm{m} \mathrm{X}$-band reflectarray, all the patch elements used here, as illustrated in Fig. 5, are circularly polarized and identical except that their angular rotations are differently oriented to achieve the required far-field co-phasal radiation [6]. This design is expected to achieve better cross-pol, sidelobe level, and radiation efficiency than the design of the $1-\mathrm{m} \mathrm{X}$-band antenna where co-phasing is achieved by using elements with different-length phase delay lines. This Ka-band reflectarray, using single-layer approach, has no membrane spacing problem like the one encountered by the X-band antenna. However, due to its higher frequency, the surface flatness will be a major concern, which will be determined primarily by the quality of the mechanical inflatable structure. 
Acknowledgment: The authors are grateful to David Cadogan, John Lin, Robert Lingo, and Charles Sandy of ILC Dover, Inc. for their development of the inflatable mechanical structure. The research described in this paper was carried out by the Jet Propulsion Laboratory, California Institute of Technology, under contract with the National Aeronautics and Space Administration.

\section{References:}

1. J. Huang, M. Lou, and E. Caro, "Super-low-mass spaceborne SAR array concepts", IEEE AP-S/URSI Symposium digest, Vol. 2, July 1997, pp. 1288-1291.

2. R. E. Munson and H. Haddad, "Microstrip reflectarray for satellite communication and RCS enhancement and reduction", U.S. Patent 4,684,952, August 1987.

3. J. Huang, "Microstrip reflectarray", IEEE AP-S/URSI Symposium digest, Vol. 2, June 1991, pp. 612-615.

4. J. Huang, "A high-gain circularly polarized Ka-band microstrip reflectarray", Microwave and Optical technology Letters, Vol. 14, No. 3, Feb. 1997, pp 163-166.

5. D. M. Pozar and T. A. Metzler, "Analysis of a reflectarray antenna using microstrip patches of variable size", Electronics Letters, April 1993, pp. 657-658.

6. J. Huang and R. J. Pogorzelski, "A Ka-band microstrip reflectarray with elements having variable rotation angles", IEEE Trans. Ant. and Propag. Vol. 46, May 1998, pp. 650-656. 


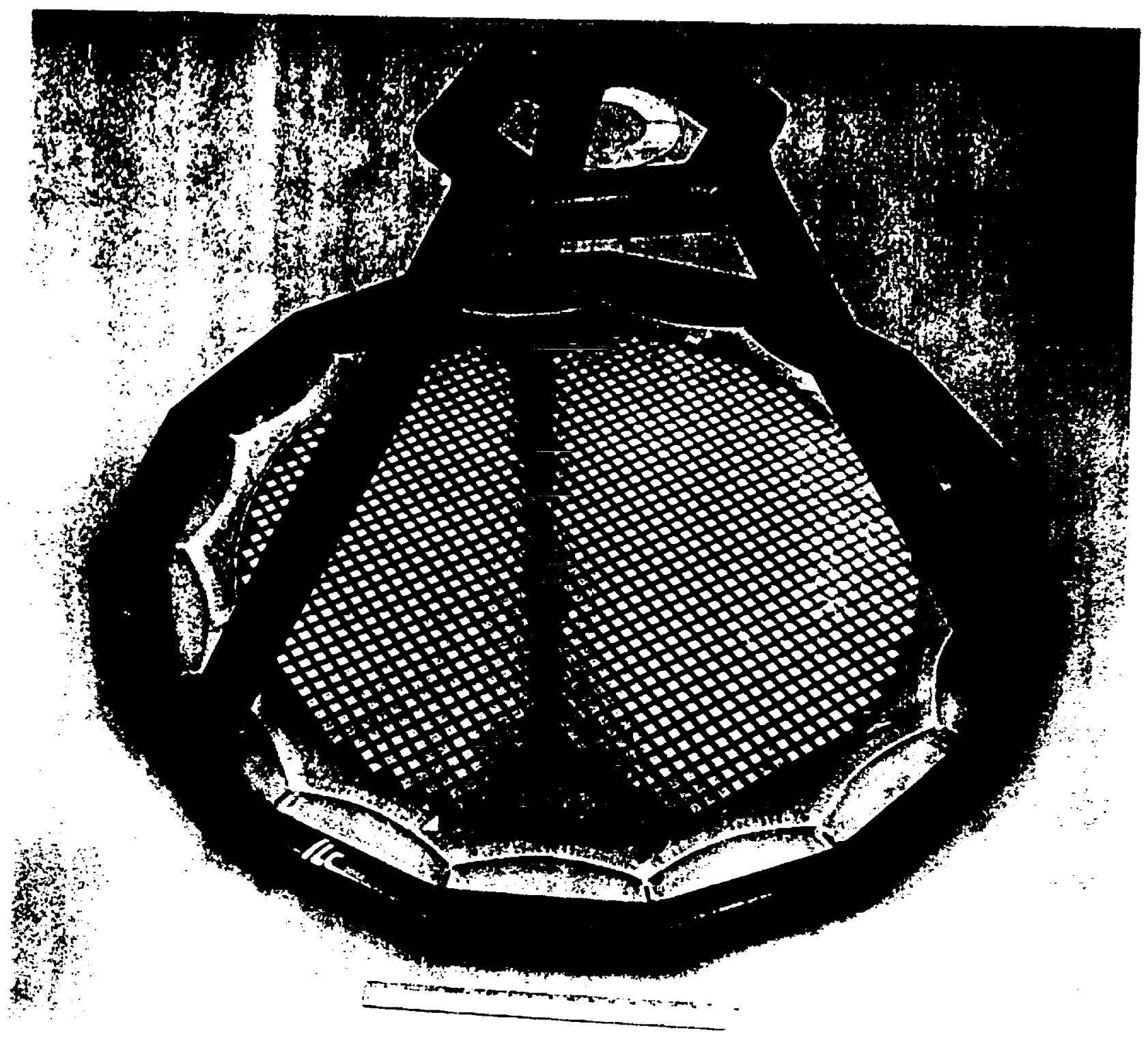

Fig. 1 Photo of the X-band 1-meter-aperture inflatable microstrip reflectarray 

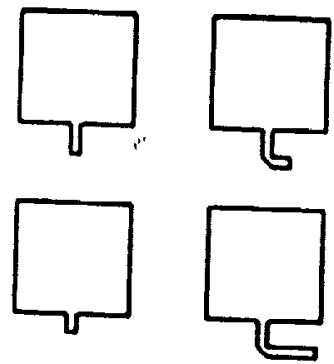

Fig. 2 Patches with variable-length phase delay lines

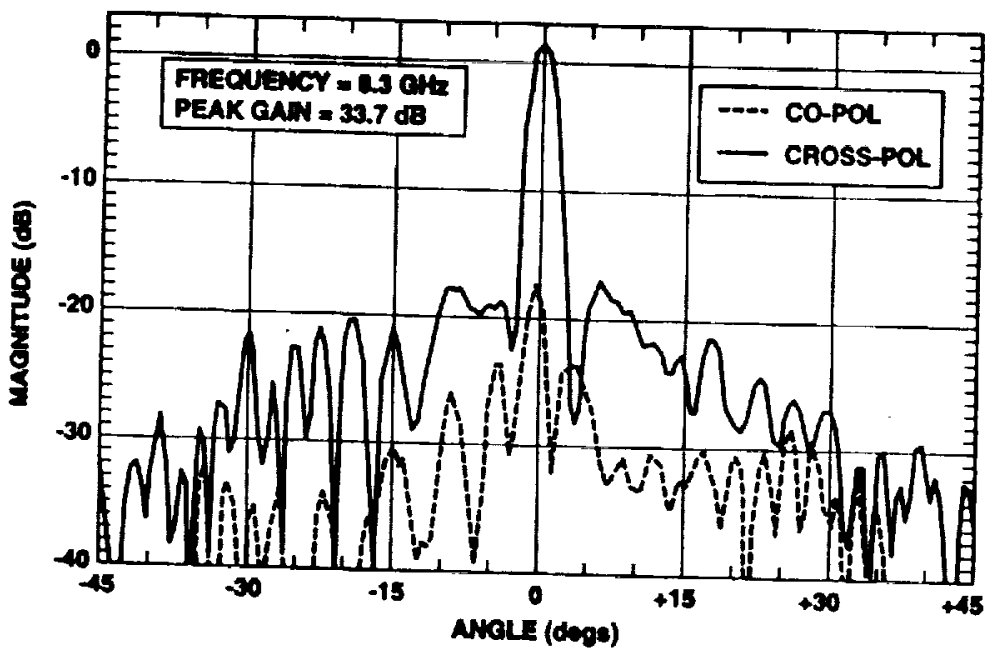

Fig. 3 Measured pattern of the X-band $1-m$ inflatable reflectarray 


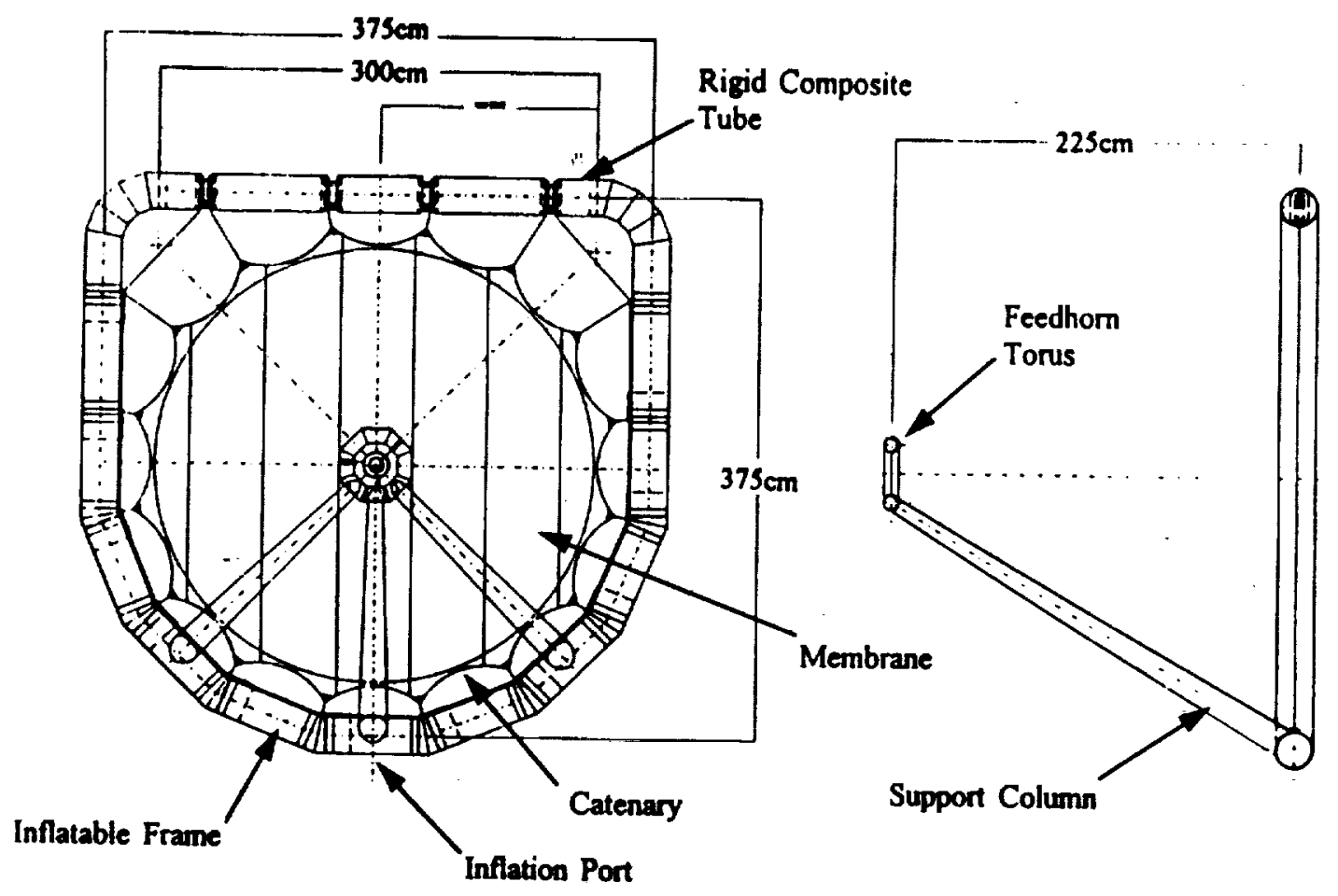

Fig. 4 Configuration of the Ka-band 3-m inflatable microstrip reflatarray<smiles>O=C1CC=CCC1</smiles>

Fig. 5. Identical circularly polarized patches with variable rotation angles 